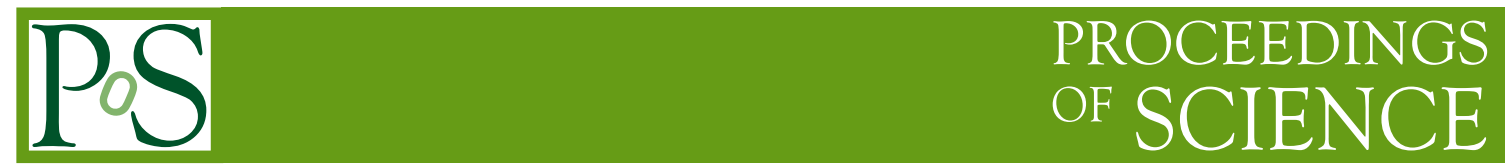

\title{
Dimuon reconstruction for B physics in ATLAS
}

\author{
Massimo Corradi* \\ INFN Bologna \\ E-mail: Massimo.Corradi@bo.infn.it
}

On behalf of the ATLAS Collaboration

The performance of the ATLAS muon reconstruction and trigger during the 2010 data taking are reviewed, with particular emphasis on the dimuon sample to be used for the ATLAS B phyiscs programme.

The 13th International Conference on B-Physics at Hadron Machines - Beauty2011, April 04-08, 2011

Amsterdam, The Netherlands

${ }^{*}$ Speaker. 


\section{Introduction}

During the year 2010 the ATLAS detector [1] has been recording $p p$ collisions at $\sqrt{s}=7 \mathrm{TeV}$ for a total integrated luminosity of $45 \mathrm{pb}^{-1}$. These data served to calibrate the ATLAS detector and to study its performance. The aim of this note is to review the performance of muon reconstruction and muon triggers, with particular attention to dimuon signatures that are the main data sample for the ATLAS B-physics programme. The first charm and beauty measurements based on the same data sample are presented in other contributions to these proceedings [2].

\section{Muon reconstruction}

Two main types of reconstructed muons are used in ATLAS for B physics analyses: combined muons (CB), in which a muon reconstructed by the Muon Spectrometer (MS) is combined with a track from the inner detector (ID), and segment-tagged muons (ST), in which a track from the ID is matched to a track segment in the MS. The second type has lower purity but recovers efficiency when the muon does not cross enough muon chambers to allow an independent reconstruction in the MS, typically because its $\mathrm{p}_{\mathrm{T}}$ is too low or because it goes through less instrumented regions at $\eta \simeq 0$ or in the barrel-endcap boundaries at $|\eta| \simeq 1-1.2$. Two independent reconstruction chains, each implementing CB and ST muons, are used in ATLAS. This redundancy allows a cross check of the performances of the reconstruction algorithms. Since the performances of the two chains are similar, only results for one of the two chains are presented here.

The performance and physics results presented in this conference are mainly based on the first-pass reconstruction, performed within a few days of data collection. The whole data set was reprocessed using updated programs and calibration constants after the end of the 2010 data taking.

The reconstruction efficiency was measured using the so-called "tag and probe" method at the $J / \psi$ resonance [3], for low- $\mathrm{p}_{\mathrm{T}}$ muons, and at the $Z$ resonance [4], for higher- $\mathrm{p}_{\mathrm{T}}$ muons. In this method muon pairs are selected by requiring a well reconstructed muon matched to a trigger
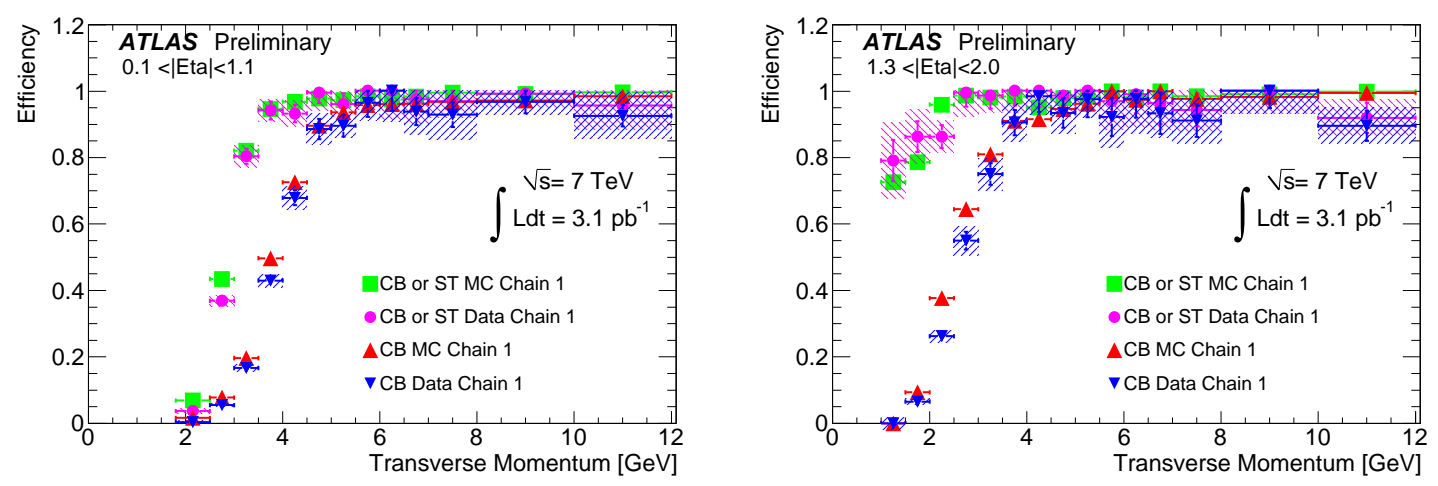

Figure 1: Muon reconstruction efficiency as a function of $\mathrm{p}_{\mathrm{T}}$ for (left) $0.1<|\eta|<1.1$ and (right) $1.3<$ $|\eta|<2.0$ [3]. The filled points correspond to data with the statistical (error bars) and systematical (shaded band) uncertainty. Empty points show the MC expectation. Triangles are for combined muons and circles for combined or segment-tagged muons (see text). 


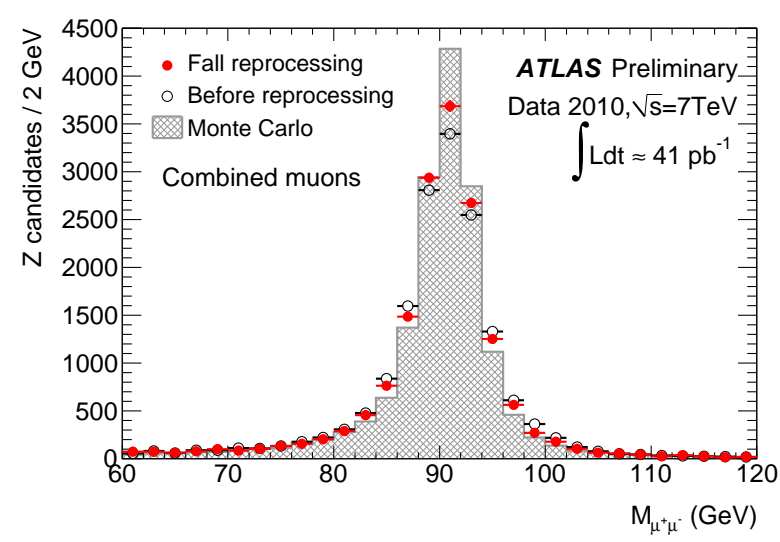

Figure 2: Dimuon invariant mass in the $Z$ region. The points show data after the first-pass reconstruction (empty circles) and after reprocessing (filled circles). The histogram shows the expected shape from a MC simulation with perfect alignment.

object, the "tag", and an inner detector track, the "probe", consistent with coming from a $\mu^{+} \mu^{-}$ resonance. In this way the "probes" are selected independently of the MS and can be used to measure the efficiency for reconstructing a muon. The subtraction of the background below the $J / \psi$ peak is important and introduces the main systematic uncertainty on the measurement. For $\mathrm{p}_{\mathrm{T}}>6 \mathrm{GeV}$ the background can be substantially reduced requiring that the muon also be identified from its energy deposition in the calorimeters. Figure 1 shows the efficiency measured at low- $\mathrm{p}_{\mathrm{T}}$, exploiting the $J / \psi$ resonance, in two $\eta$ regions. The plateau for CB muons starts at $\mathrm{p}_{\mathrm{T}}=5$ (4) GeV in the barrel (endcap) region. When ST muons are added, the beginning of the plateau is shifted to lower $\mathrm{p}_{\mathrm{T}}$. The efficiency at plateau is about $98 \%$ for $|\eta|<2.5$ when ST muons are included. The measurement based on the $J / \psi$ resonance is in good agreement with the precise results obtained at high- $\mathrm{p}_{\mathrm{T}}$ with the $Z$. The MC describes the data well, with an overall data/MC scale factor at high $\mathrm{p}_{\mathrm{T}}$ of $0.9806 \pm 0.0031$ for $\mathrm{CB}$ and $0.9990 \pm 0.0016$ for $\mathrm{CB}+\mathrm{ST}$ muons, with an additional systematic uncertainty of \pm 0.002 .

A relevant figure to assess the background rejection capabilities of the muon reconstruction is the probability that a pion is reconstructed as a muon. This "fake probability" was measured using identified pions from $K_{S}^{0}$ decays [5]. For CB plus ST muons with $p_{T}>4 \mathrm{GeV}$ the fake probability is $(0.248 \pm 0.047) \%$, in agreement with the MC expectation.

The muon momentum scale and resolution was studied with cosmic rays [6], from the width of the $Z$ mass peak, and from the comparison of the independent momentum measurements provided by the ID and the MS [ [7]. Figure 2 shows the dimuon invariant mass at the $Z$ resonance from the first-pass reconstruction and after reprocessing, compared to a $\mathrm{MC}$ with perfect alignment. The agreement is satisfactory although the width in data is still larger than in the perfectly aligned MC. The main differences in resolution between data and $\mathrm{MC}$ are related to the ID resolution at large $|\eta|$, where the alignment is not yet final. 

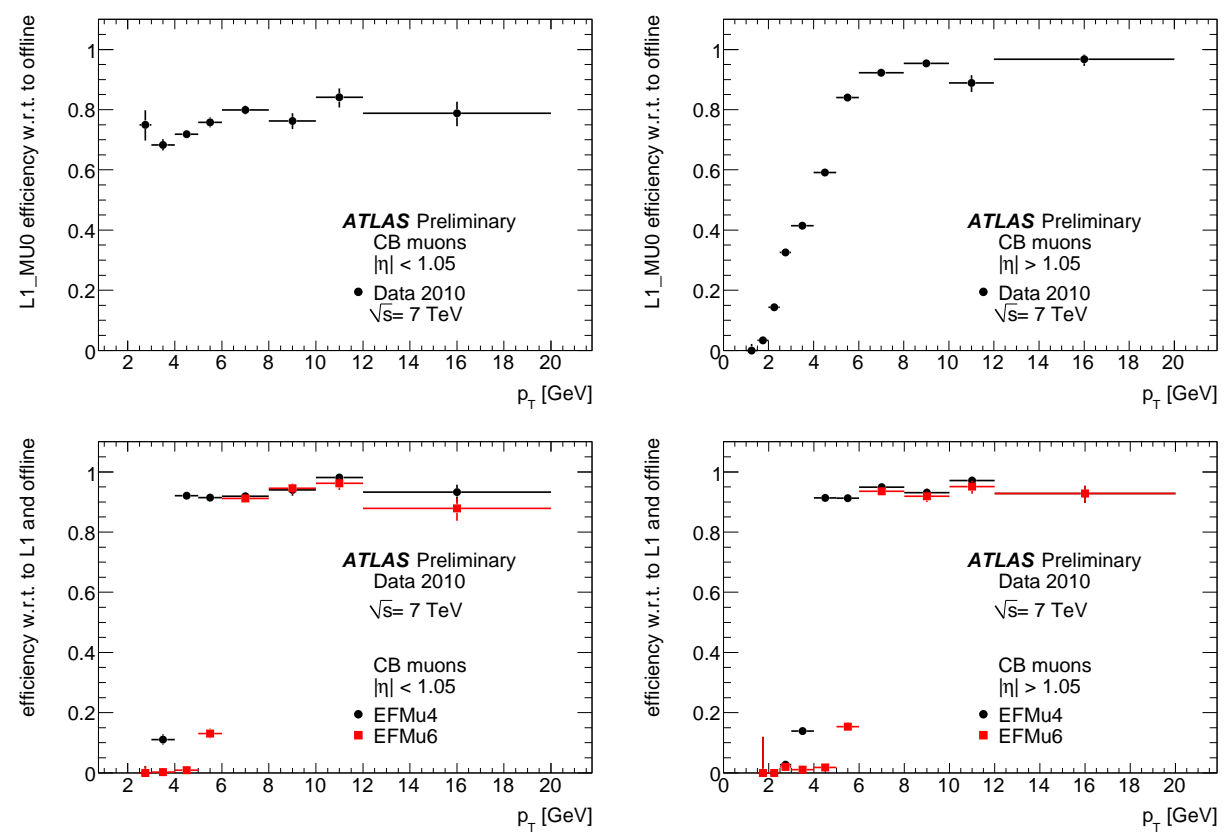

Figure 3: Trigger efficiencies as a function of muon $\mathrm{p}_{\mathrm{T}}$ in the barrel (left) and endcaps (right) [3]. Upper plots: level one efficiency for offline combined muons. Lower plots: efficiency of the higher level trigger (L2 and EF) for offline combined muons triggered by level one. Two different $\mathrm{p}_{\mathrm{T}}$ thresholds are shown for the higher level trigger: $4 \mathrm{GeV}$ (EFMu4) and $6 \mathrm{GeV}$ (EFMu6).

\section{Dimuon triggers}

The ATLAS trigger is built in three levels. For muons, level one (L1) is based on dedicated hardware that checks for coincidences in time and space between different stations of trigger detectors: RPCs in the barrel $(|\eta|<1.05)$ and TGCs in the endcaps $(1.05<|\eta|<2.4)$. At level two (L2) the MS and ID data from a region of interest identified by L1 are used by a fast muon reconstruction algorithm. In the third level, called the Event Filter (EF), the whole event is available and algorithms similar to the offline reconstruction are used. In 2010, the ATLAS trigger was running at an average output rate of $\approx 200 \mathrm{~Hz}$, mainly limited by the available offline resources. A fraction of the total bandwidth between 10 and $20 \%$ was assigned to muon and B-physics triggers. As the luminosity increased during the data taking period, reaching $2 \times 10^{32} \mathrm{~cm}^{-2} \mathrm{~s}^{-1}$, higher- $\mathrm{p}_{\mathrm{T}}$ thresholds were applied to single-muon triggers. Most of the integrated luminosity was collected with a lowest unprescaled single-muon threshold $\mathrm{p}_{\mathrm{T}}>13 \mathrm{GeV}$. The $J / \psi$ cross section measurement [8] is based on an initial sample of $2.3 \mathrm{pb}^{-1}$ in which single-muon triggers with $\mathrm{p}_{\mathrm{T}}$ thresholds of 4 or $6 \mathrm{GeV}$ were still taking data without prescale.

To collect a large sample of B decays, different types of dimuon triggers were deployed to recover low- $\mathrm{p}_{\mathrm{T}}$ dimuon pairs that could not be recorded with single-muon triggers. Standard dimuon triggers, requiring two muon triggers in the same event, had a lowest unprescaled $\mathrm{p}_{\mathrm{T}}$ threshold of $6 \mathrm{GeV}$ per muon. Triggers dedicated to B physics, with the additional requirements that the two muons had opposite charge, matched to a common vertex, and that the invariant mass was larger 


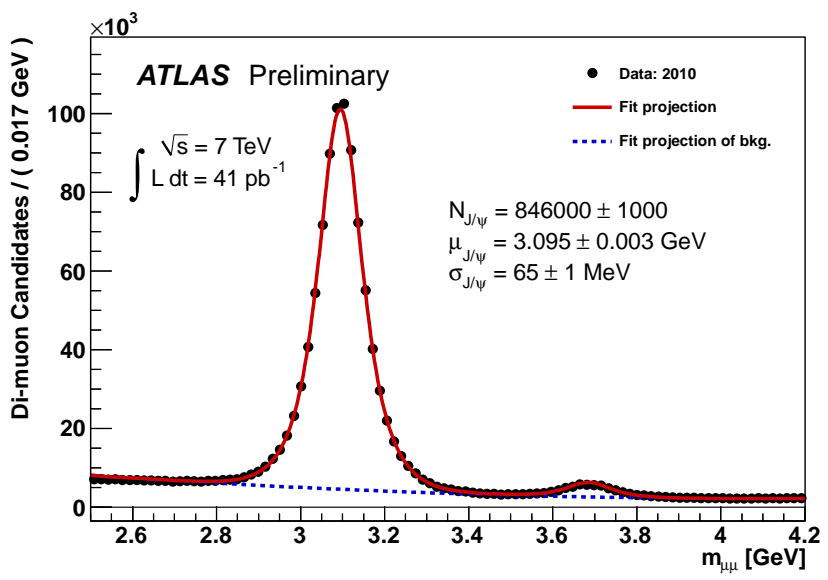

Figure 4: Dimuon invariant mass for the full 2010 data sample in the $J / \psi$ region.
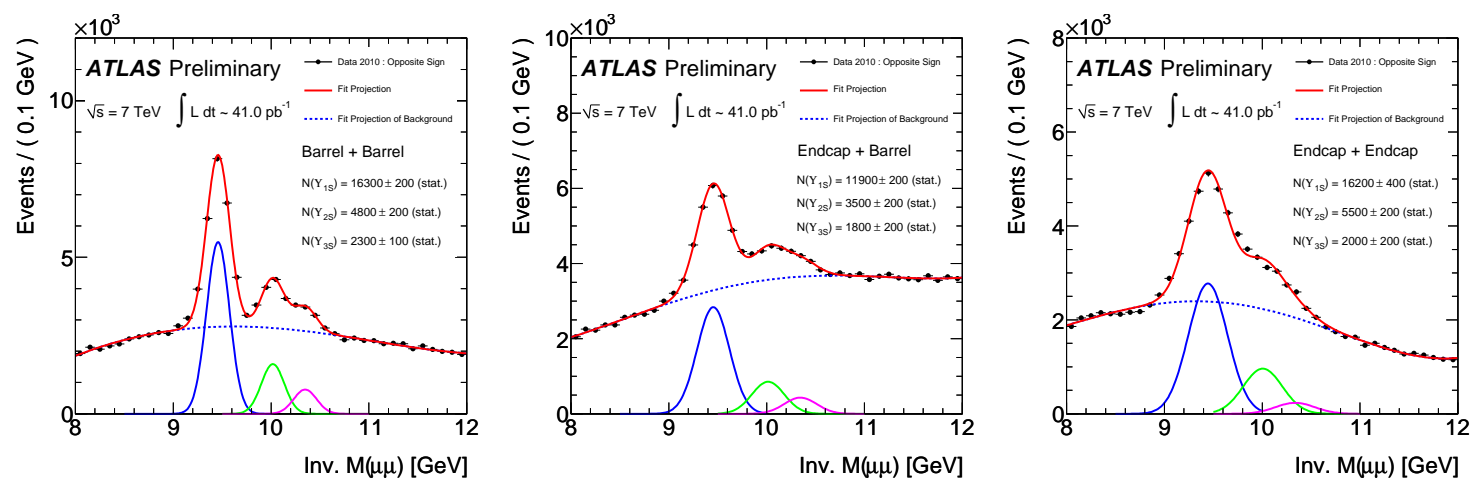

Figure 5: Dimuon invariant mass in the $\Upsilon$ region for events with both muons in the barrel (left), one in the barrel and one in the endcaps (center) and both in the endcaps (right).

than $500 \mathrm{MeV}$, collected pairs with $\mathrm{p}_{\mathrm{T}}>4 \mathrm{GeV}$ for each muon during the whole period. In addition it was possible to run dedicated trigger chains seeded by a single-muon L1 trigger in which a second low- $\mathrm{p}_{\mathrm{T}}$ muon was searched for in $\mathrm{L} 2$ and $\mathrm{EF}$, increasing significantly the acceptance at low $\mathrm{p}_{\mathrm{T}}$. With these requirements a good acceptance was obtained for the $\Upsilon$ resonances down to $p_{\mathrm{T}}(\Upsilon)=0$ while the acceptance for the $J / \psi$ was limited to $p_{\mathrm{T}}(J / \psi)>6(1) \mathrm{GeV}$ in the barrel (endcaps).

More details on the muon trigger performances with early 2010 data are reported in [9]. Figure 3 shows the trigger efficiency at low- $\mathrm{p}_{\mathrm{T}}$ for L1 and the combined efficiency of L2 and EF, measured using the "tag and probe" method at the $J / \psi$. The plateau efficiency for L1 in the barrel is $\approx 82 \%$, mainly due to limitations in the geometrical acceptance related to the supports of the ATLAS detectors and to services, while it is close to $95 \%$ in the endcaps. The L2 and EF efficiency at plateau is above $90 \%$. 

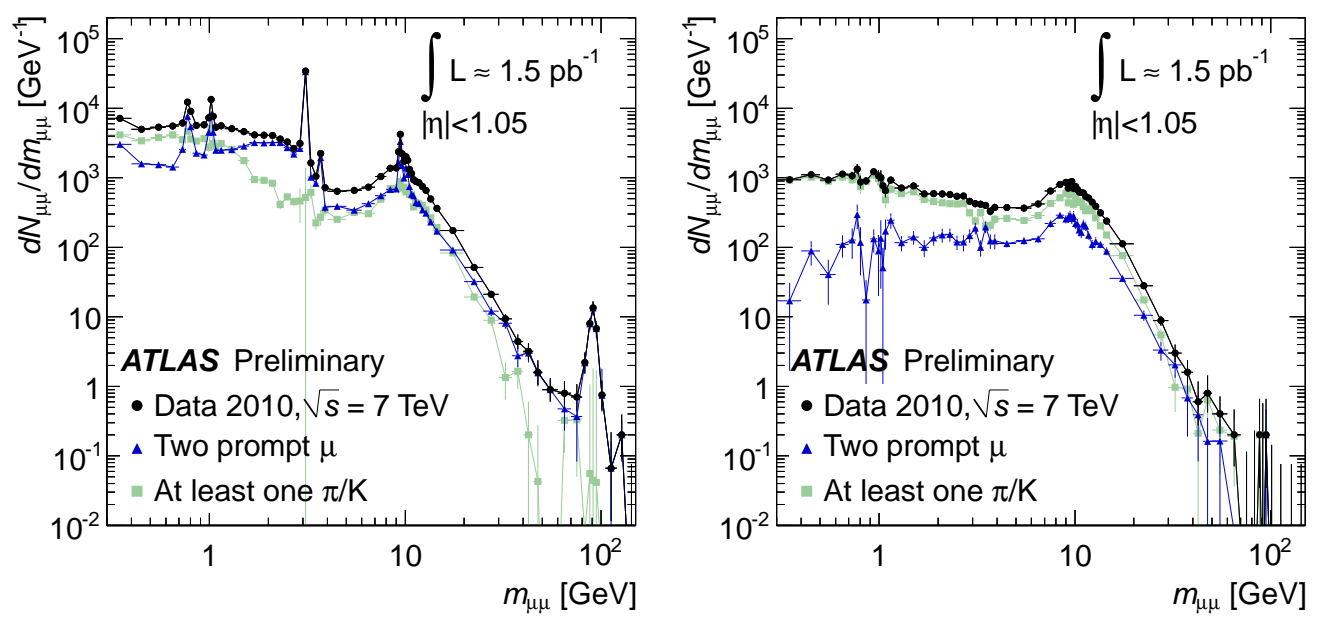

Figure 6: Decomposition of the dimuon invariant mass spectrum into a prompt and a non-prompt component from $\pi, K$ decays and punch through [10]. Left: opposite charge dimuons, right: same charge dimuons.

\section{Dimuon samples}

Figure $\emptyset$ shows the dimuon invariant mass around the $J / \psi$ mass based on the full sample collected in 2010 using single- and di-muon triggers. The selection requires two opposite-charge muons matched to the same vertex. One is required to be a $\mathrm{CB}$ muon with $\mathrm{p}_{\mathrm{T}}>4 \mathrm{GeV}$ and the other to be a CB or a ST muon with $\mathrm{p}_{\mathrm{T}}>2.5 \mathrm{GeV}$. The signal from the fit corresponds to $846 \mathrm{k} J / \psi$. The peak position is $3.095 \mathrm{GeV}$, in agreement with the PDG value within the $0.003 \mathrm{GeV}$ uncertainty from the momentum scale. The $\psi(2 S)$ peak is also apparent. Figure 5 shows the invariant mass region of the $\Upsilon$ resonances. To account for the dependence of the signal resolution on the event topology, the distribution is shown separately for three cases: both muons in the barrel, one in the barrel and one in the endcaps and both in the endcaps. The fit is performed with three Gaussians with fixed mass differences plus a polynomial background. The total $\Upsilon(1 S)$ signal consists of $44 \mathrm{k}$ events.

The dimuon sample is composed of Drell-Yan pairs, muons from $\mathrm{c}$ and $\mathrm{b}$ decays, and a sizable fraction of pairs in which at least one of the two muons is a non-prompt muon, originating from in-flight decays of pions or kaons or from hadronic punch through. It is possible to distinguish nonprompt muons on a statistical basis by searching for a kink in the muon track in the ID, which would indicate a $\pi$ or $K$ decay inside the inner detector. The difference between the momentum measured in the ID and in the MS provides another tool to distinguish late $\pi$ and $K$ decays and punch-through muons from the prompt component. A combined discriminant, based on the probability associated to the largest scatter in the ID and on the relative momentum mismatch between MS and ID, was used to study the non-prompt content of a dimuon sample [10]. The sample was selected using early 2010 data in which a single-muon trigger with a $\mathrm{p}_{\mathrm{T}}$ threshold of $4 \mathrm{GeV}$ was active, requiring two opposite-charge $\mathrm{CB}$ muons, one with $\mathrm{p}_{\mathrm{T}}>4 \mathrm{GeV}$ and one with $\mathrm{p}_{\mathrm{T}}>3 \mathrm{GeV}$, both with $|\eta|<1.05$. The fraction of pairs in wich at least one muon is non-prompt is $(31 \pm 4) \%$. The invariant mass distribution is shown in Figure 6 with the prompt and non-prompt components superimposed. Non- 


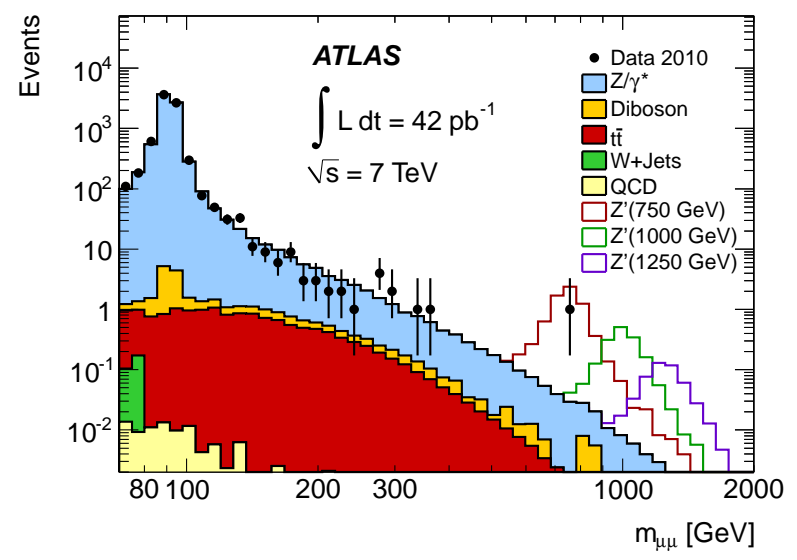

Figure 7: High mass dimuon spectrum. The 2010 data are compared to the Standard Model expectations and to the $Z^{\prime}$ signal for three different masses [11].

prompt muons dominate at masses below $2 \mathrm{GeV}$, are a few percent of the total in the $J / \psi$ region, and $\sim 30 \%$ for $m>20 \mathrm{GeV}$. Harder cuts on the quality of the ID track and of the match between ID and MS can be used to further reduce the non-prompt muon contamination.

This overview of the dimuon spectrum ends with a look at its high-mass tail, Figure 7. Candidates are observed up to a mass of $768 \mathrm{GeV}$. The invariant mass distribution is in good agreement with the Standard Model. The limit on a hypothetical $Z^{\prime}$ boson, obtained from the muon channel, is $m\left(Z^{\prime}\right)>834 \mathrm{GeV}$ (assuming the couplings of the Sequential Standard Model) [11].

\section{Conclusions}

During the first year of data taking at $\sqrt{s}=7 \mathrm{TeV}$ the ATLAS detector collected a large sample of muons, which allowed a detailed study and tune the muon reconstruction performances and of the muon triggers. The measured reconstruction efficiency at plateau is around $98 \%$, in agreement with the $\mathrm{MC}$ expectation. The dimuon triggers devised for B physics also performed well, providing a sample of $850 \mathrm{k} J / \psi$ and $44 \mathrm{k} \Upsilon(1 S)$. At the time of writing these proceedings in June 2011, ATLAS has collected about $1 \mathrm{fb}^{-1}$.

\section{References}

[1] ATLAS Collaboration, JINST 3 (2008) S08003.

[2] I. Christidi, Open $b$ and $\bar{b}$ cross sections using inclusive and exclusive channels at ATLAS and Shih-Chien Hsu, $J / \psi$ production in $p-p$ and in heavy ions in ATLAS, these proceedings.

[3] ATLAS Collaboration, ATLAS-CONF-2011-021, http://cdsweb.cern.ch/record/1336750.

[4] ATLAS Collaboration, ATLAS-CONF-2011-008, http://cdsweb.cern.ch/record/1330715; ATLAS Collaboration, ATLAS-CONF-2011-063, http://cdsweb.cern.ch/record/1345743.

[5] ATLAS Collaboration, ATLAS-CONF-2010-064, http://cdsweb.cern.ch/record/1281339. 
[6] ATLAS Collaboration, Eur. Phys. J. C 71 (2011) 1593 [arXiv:1011.6665].

[7] ATLAS Collaboration, ATLAS-CONF-2011-046,http://cdsweb.cern. ch/record/1338575.

[8] ATLAS Collaboration, accepted by Nucl. Phys. B [arXiv:1104.3038].

[9] ATLAS collaboration, ATLAS-CONF-2010-095, http://cdsweb.cern.ch/record/1299573.

[10] ATLAS Collaboration ATLAS-CONF-2011-003, http: //cdsweb.cern. ch/record/1326960.

[11] ATLAS Collaboration, Phys. Lett. B 700 (2011) 163-180 [arXiv:1103.6218]. 